\title{
Discrete Multiobjective Optimization
}

\author{
Kathrin Klamroth \\ University of Wuppertal, 42097 Wuppertal, Germany \\ klamroth@math.uni-wuppertal.de \\ http://www . math.uni-wuppertal.de/ ${ }^{k}$ lamroth
}

\section{Problems and Applications}

Multiple objective combinatorial optimization (MOCO) has become a quickly growing field in multiple objective optimization, and has recently attracted the attention of researchers both from the fields of multiple objective optimization and from single objective integer programming. Typical examples of MOCO problems include multiple objective knapsack problems with applications, among others, in capital budgeting, multiple objective assignment problems, the multiple objective traveling salesman problem, and other network problems like multiple objective minimum spanning tree, shortest path, and minimum cost flow problems. Formally, a general MOCO problem can be stated as $\min \left\{f(x)=\left(f_{1}(x), \ldots, f_{p}(x)\right)^{T}: x \in X\right\}$, where the decision space $X$ is a given discrete feasible set that usually has some additional combinatorial structure.

Since the set of feasible solutions of a MOCO problem is discrete and usually finite, it can at least theoretically be enumerated to identify all Pareto optimal solutions. This is, however, generally impractical due to the exponentially growing number of feasible (and sometimes also Pareto optimal) solutions.

\section{Neighborhood Search and Connectedness}

Structural properties of the efficient set of MOCO problems play a crucial role for the development of efficient solution methods. A central question in the context of metaheuristics relates to the connectedness of the efficient set with respect to combinatorially or topologically motivated neighborhood structures. A positive answer to this question would provide a theoretical justification for the application of fast neighborhood search techniques, not only for multiple objective but also for appropriate formulations of single objective problems.

Unfortunately, most of the classical MOCO problems do in general not possess a connectedness property with respect to reasonable neighborhoods. This includes, among others, knapsack problems (and even several special cases of knapsack problems) and linear assignment problems, shortest path problems as well as spanning tree and minimum cost flow problems. Numerical tests performed for different variants of the knapsack problem suggest, however, that the likelihood with which non-connected adjacency graphs occur in randomly generated problem instances is very small. 